\title{
PRODUCT INTEGRAL TECHNIQUES FOR ABSTRACT HYPERBOLIC PARTIAL DIFFERENTIAL EQUATIONS $\left({ }^{1}\right)$
}

BY

\section{J. W. SPELLMANN}

\begin{abstract}
Explicit and implicit product integral techniques are used to represent a solution $U$ to the abstract system: $U_{12}(x, y)=A U(x, y) ; U(x, 0)=$ $p=U(0, y)$. The coefficient $A$ is a closed linear transformation defined on a dense subspace $D(A)$ of the Banach space $X$ and the point $p$ in $D(A)$ satisfies the condition that $\left\|A^{i} p\right\|<S^{i}(i \text { !) })^{3 / 2}$ for all integers $i \geqslant 0$ and some $S>0$. The implicit technique is developed under the additional assumption that $A$ generates a strongly continuous semigroup of bounded linear transformations on $X$. Both methods provide representations for the $J_{0}$ Bessel function.
\end{abstract}

I. Introduction. Let $X$ denote a Banach space and $A$ denote a closed linear (and, in general, unbounded) transformation defined on a dense subspace $D(A)$ of $X$. A point $p$ in $X$ is said to satisfy condition (I) if and only if

(a) $p$ is in the domain of $A^{i}$ for all positive integers $i$ and

(b) there is a positive number $S$ such that $\left\|A^{i} p\right\|<S^{i}(i !)^{3 / 2}$ for all integers $i \geqslant 0$.

In this paper two product integral type techniques are used to represent a solution to

$$
U_{12}(x, y)=A U(x, y), \quad U(x, 0)=p=U(0, y),
$$

where $U$ is a function from the unit disk $[0,1] \times[0,1]$ to $D(A)$ and $p$ is a point in $X$ satisfying condition (I).

In §II an explicit product integral type method is used to represent a solution to system (II). The additional assumption that $A$ is the infinitesimal generator of a strongly continuous semigroup of bounded linear transformations is used in §III to develop an implicit product integral type representation of a solution

Received by the editors March 5, 1974 and, in revised form, May 30, 1974. AMS (MOS) subject classifications (1970). Primary 47F05; Secondary 33A40.

Key words and phrases. Product integration, linear semigroups of operators, abstract partial differential equations, $J_{0}$ Bessel function.

${ }^{1}$ ) This project was funded by the Pan American University Faculty Research Council, Grant \#1521. 
to system (II). The results of $\S \S I$ II and III are used in $\S I V$ to produce representations for the $J_{0}$ Bessel function. In $\S \mathrm{V}$ the product integral representations developed in $\S \S I I$ and III are used in an example. A second example is given in $\S \mathrm{V}$ of a case where Theorem 1 applies but Theorem 2 does not.

In 1958, J. B. Diaz published (in [3]) an Euler-Cauchy polygon technique for the real partial differential system

$$
\begin{aligned}
& U(x, y)=f\left(x, y, U(x, y), U_{1}(x, y), U_{2}(x, y)\right), \\
& U(x, 0)=g(x), \quad U(0, y)=h(y),
\end{aligned}
$$

where $f$ is considered continuous, bounded and satisfies a Lipschitz condition. The boundary functions, $g$ and $h$, are assumed to be continuously differentiable. Similar work by I. I. Glick appeared subsequently in [5] for the higher order equation $U x_{1} \ldots x_{n}=f$. Recently, Lovelady announced (in [7]) results concerning the existence and uniqueness of solutions to a Banach space analog of system (III) where $f$ is assumed to have a continuous Fréchet derivative. In [8], this author showed that condition (I) is satisfied by all points in a dense subset of $X$ whenever $A$ is the infinitesimal generator of a strongly continuous semigroup of bounded linear transformations. Hasegawa in [6] and Chernoff in [2] have also investigated sets of vectors which satisfy properties similar to condition (I).

The author would like to express his appreciation for the suggestions of the referee, especially the Corollary to Theorem 2 and the interesting second example of $\S \mathrm{V}$.

II. An explicit product integral representation. In this section a solution to system (II) will be represented as the uniform limit of a sequence of explicit product integral type approximations. The explicit technique for system (II) is developed by considering the following integral equation:

$$
U(x, y)=U(x, b)+U(a, y)-U(a, b)+\int_{a}^{x} \int_{b}^{y} A U(s, t) d t d s
$$

along with the boundary condition

$$
U(x, 0)=p=U(0, y)
$$

where $U$ is a function from $[0,1] \times[0,1]$ to $D(A)$ satisfying (1) and (2) for each pair of points, $(x, y)$ and $(a, b)$, such that $0 \leqslant a \leqslant x \leqslant 1$ and $0 \leqslant b \leqslant$ $y \leqslant 1$; and $p$ is a point in $D(A)$ satisfying condition (I). Replacing the integral in equation (1) by 


$$
(x-a)(y-b) \mathbf{A}[U(x, b)+U(a, y)-U(a, b)]
$$

yields the following approximation for $U(x, y)$ :

$$
U(x, y) \approx(I+(x-a)(y-b) A)[U(x, b)+U(a, y)-U(a, b)] .
$$

It is noted that repeated use of (3) over a grid of the rectangle $[(0,0) ;(x, y)]$ will yield an approximation of $U(x, y)$ in terms of the boundary conditions on the $x$ and $y$ axis prescribed by (2). From this fact a sequence of approximating functions will be constructed.

For each point $(x, y)$ in $[0,1] \times[0,1]$ and each pair $(k, l)$ of positive integers, let

$$
x_{i k}=(i x) / k \text { for } i=0,1, \ldots, k \text {, }
$$

$$
y_{j l}=(j y) / l \text { for } j=0,1, \ldots, l
$$

and

$$
G_{k l}^{x y}=\left\{\left(x_{i k}, y_{j l}\right) \mid i=0,1, \ldots, k ; j=0,1, \ldots, l\right\}
$$

Using the same restrictions on the point $(x, y)$ and the pair $(k, l)$ of integers, let $\left[V_{k l}^{x y}\right]$ denote a function motivated by (3) and defined on the grid $G_{k l}^{x y}$ by

$$
\left[V_{k l}^{x y}\right]\left(x_{i k}, y_{j l}\right)= \begin{cases}p & \text { if } x_{i k} y_{j l}=0, \\ {\left[B_{k l}^{x y}\right]\left[\Delta V_{k l}^{x y}\right]\left(x_{i k}, y_{j l}\right)} & \text { if } x_{i k} y_{j k}>0,\end{cases}
$$

where

$$
\left[B_{k l}^{x y}\right]=(I+(x y / k l) A)
$$

and

$$
\left[\Delta V_{k l}^{x y}\right]\left(x_{i k}, y_{j l}\right)=\left[V_{k l}^{x y}\right]\left(x_{i-1 k}, y_{j l}\right)+\left[V_{k l}^{x y}\right]\left(x_{i k}, y_{j-1 l}\right)
$$

$$
-\left[V_{k l}^{x y}\right]\left(x_{i-1 k}, y_{j-1 k}\right) .
$$

An induction argument may be used to establish that each function $\left[V_{k l}^{x y}\right]$ may be defined in terms of the constant boundary value $p$ by 


$$
\begin{aligned}
{\left[V_{x y}^{k l}\right](x, y)=} & {\left[V_{x y}^{k l}\right]\left(x_{k k}, y_{l l}\right) } \\
= & \sum_{i=0}^{l-1}\left(\begin{array}{c}
k-1+i \\
k-1
\end{array}\right) B^{k+i} \sum_{j=0}^{(l-1)-i}(-1)^{j}\left(\begin{array}{l}
k \\
j
\end{array}\right) p \\
& +\sum_{i=0}^{l-1}\left(\begin{array}{c}
k-1+i \\
l-1
\end{array}\right) B^{k+i} \sum_{j=0}^{(l-1)-i}(-1)^{j}\left(\begin{array}{l}
l \\
j
\end{array}\right) p \\
& +\sum_{i=0}^{l-1}\left(\begin{array}{c}
l-1 \\
i
\end{array}\right)\left(\begin{array}{c}
k-1+i \\
l-1
\end{array}\right)(-1)^{l+i} B^{k+i} p \\
& +\sum_{i=0}^{k-l-1}\left(\begin{array}{c}
l-1+i \\
l-1
\end{array}\right) B^{l+i} \sum_{j=0}^{l}(-1)^{j}\left(\begin{array}{l}
l \\
j
\end{array}\right) p
\end{aligned}
$$

for $k \geqslant l$ and that

$$
\begin{aligned}
{\left[V_{k l}^{x y}\right](x, y)=} & {\left[V_{k l}^{x y}\right]\left(x_{k k}, y_{l l}\right) } \\
= & \sum_{i=0}^{k-1}\left(\begin{array}{c}
l-1+i \\
l-1
\end{array}\right) B^{l+i} \sum_{j=0}^{(k-1)-i}(-1)^{j}\left(\begin{array}{l}
l \\
j
\end{array}\right) p \\
& +\sum_{i=0}^{k-1}\left(\begin{array}{c}
l-1+i \\
k-1
\end{array}\right) B^{l+i} \sum_{j=0}^{(k-1)-i}(-1)^{j}\left(\begin{array}{l}
k \\
j
\end{array}\right) p \\
& +\sum_{i=0}^{k-1}\left(\begin{array}{c}
k-1 \\
i
\end{array}\right)\left(\begin{array}{c}
l-1+i \\
k-1
\end{array}\right)(-1)^{k+i} B^{l+i} p \\
& +\sum_{i=0}^{l-k-1}\left(\begin{array}{c}
k-1+i \\
k-1
\end{array}\right) B^{k+i} \sum_{j=0}^{k}(-1)^{j}\left(\begin{array}{l}
k \\
j
\end{array}\right) p
\end{aligned}
$$

for $l \geqslant k$. In equations (10) and (11), B denotes $\left[B_{k l}^{x y}\right]=(I+(x y / k l) A)$ and $\Sigma_{i=0}^{-1}$ is considered to equal 0 when $k=l$.

For each positive integer $n$, let $U_{n}$ denote the function from $[0,1] \times[0,1]$ to $X$ which is defined by

$$
U_{n}(x, y)=\left[V_{n n}^{x y}\right](x, y)
$$

By letting $k=l=n$, equation (7) (or (8)) may be reduced to give 


$$
U_{n}(x, y)=\sum_{i=0}^{n-1}(-1)^{n-1-i}\left(\begin{array}{c}
n-1 \\
i
\end{array}\right)\left(\begin{array}{c}
n-1+i \\
i
\end{array}\right)\left(I+\left(\frac{x y}{n^{2}}\right) A\right)^{n+i} p
$$

for each point $(x, y)$ in $[0,1] \times[0,1]$.

Theorem 1 shows that a solution to system (I) may be represented as the uniform limit of a sequence of explicit product integral type approximations defined by equation (13).

THEOREM 1. Suppose $A$ is a closed linear transformation defined on a dense subspace $D(A)$ of the Banach space $X$ and $p$ is a point in $X$ satisfying condition (I). For each positive integer $n$, let $U_{n}$ be the function defined on $[0,1] \times[0,1]$ by equation (13). Then the sequence $\left\{U_{n}\right\}_{n=1}^{\infty}$ of functions converges uniformly on $[0,1] \times[0,1]$ to a solution of system (II).

An examination of the proof of Theorem 8 of [8] shows that the function $U$ defined on $[0,1] \times[0,1]$ by

$$
U(x, y)=p+\sum_{i=1}^{\infty}(x y)^{i}(i !)^{-2} A^{i} p
$$

will be a solution to system (II) of this paper whenever the operator $A$ is closed and linear and that the point $p$ satisfies condition (I). Although these conditions are met by the hypothesis of Theorem 1 of this paper, they would also be satisfied in the case where $A$ generates a strongly continuous semigroup of bounded linear transformations, as was assumed in [8]. It may also be noted that if $A$ is as in [8], Theorem 7 of the same paper implies that the set of points satisfying condition (I) will be dense in $X$.

The following lemmas will be used to prove Theorem 1 by showing that the sequence $\left\{U_{n}\right\}_{n=1}^{\infty}$ converges uniformly on $[0,1] \times[0,1]$ to the function $U$ defined by (14).

LEMMA 1. Suppose that $A$ is a closed linear operator defined on a dense subspace $D(A)$ of $X$ and $p$ is a point in $D(A)$ which satisfies condition (I). Then for each number $\epsilon>0$ and each integer $i \geqslant 0$, there is a positive integer $N$ such that

$$
\left\|\left(I+\left(x y / n^{2}\right) A\right)^{n} A^{i} p-A^{i} p\right\|<\epsilon
$$

for all integers $n>N$ and all points $(x, y)$ in $[0,1] \times[0,1]$.

Proof. The proof of Lemma 1 follows from the fact that for each integer 
$i \geqslant 0$ and each point $(x, y)$ in $[0,1] \times[0,1]$ one has that

$$
\begin{aligned}
\left\|\left(I+\left(x y / n^{2}\right) A\right)^{n} A^{i} p-A^{i} p\right\| & \leqslant \sum_{j=1}^{n} n^{-2 j}\left(\begin{array}{l}
n \\
j
\end{array}\right)\left\|A^{i+j} p\right\| \\
& \leqslant \frac{1}{n} \sum_{j=1}^{n}(j !)^{-2} S^{i+j}[(i+j) !]^{3 / 2}
\end{aligned}
$$

where $S$ is defined in condition (I).

LEMma 2. Suppose the operator $A$ and the point $p$ satisfy the hypothesis of Lemma 1.

$$
\lim _{n \rightarrow \infty} \frac{1}{n} \sum_{i=0}^{n} \sum_{j=0}^{n}(i !)^{-2}(j !)^{-2}\left\|A^{i+j} p\right\|=0
$$

Proof. The proof of Lemma 2 follows from the assumption that the point $p$ satisfies condition (I).

Proof of Theorem 1. Let $\epsilon>0$. Since the point $p$ satisfies condition (I) there is a positive integer $N_{1}$ such that

$$
\sum_{i=n}^{\infty}(i !)^{-2}\left\|A^{i} p\right\|<\frac{\epsilon}{8}
$$

and

$$
\frac{1}{n} \sum_{i=0}^{n} \sum_{j=0}^{n}(i !)^{-2}(j !)^{-2}\left\|A^{i+j} p\right\|<\frac{\epsilon}{4}
$$

whenever $n>N_{1}$. Lemma 2 implies the existence of a positive integer $N_{2}$ such that

$$
\left\|\left(I+\left(x y / n^{2}\right) A\right)^{n} A^{i} p-A^{i} p\right\|<\epsilon / 12
$$

for all integers $n \geqslant N_{2}$, all integers $i$ in $\left[0, N_{1}\right]$ and all points $(x, y)$ in $[0,1] \times$ $[0,1]$. Moreover, $N_{2}$ is chosen such that

$$
\left|1-\left(1-\left(N_{1} / n\right)\right)^{N_{1}}\right|<\epsilon /(4 \Delta+4)
$$

for all integers $n>N_{2}$ where $\Delta=\Sigma_{i=0}^{N_{1}}(i !)^{-2}\left\|A^{i} p\right\|$. Let $N=\max \left\{N_{1}+1, N_{2}\right\}$. Suppose $n$ is an integer greater than $N$ and $(x, y)$ is a point in $[0,1] \times[0,1]$. A 
rearrangement of equation (13) yields that

$$
U_{n}(x, y)=\sum_{i=0}^{n-1}\left(\begin{array}{c}
n-1 \\
i
\end{array}\right)\left(\begin{array}{c}
n-1+i \\
i
\end{array}\right)\left(\frac{x y}{n^{2}}\right)^{i}\left(I+\left(\frac{x y}{n^{2}}\right) A\right)^{n} A^{i} p
$$

Letting the function $U$ be defined by equation (14), one then has that

$$
\begin{aligned}
& \left\|U_{n}(x, y)-U(x, y)\right\| \\
& =\left\|\sum_{i=0}^{n-1}\left(\begin{array}{c}
n-1 \\
i
\end{array}\right)\left(\begin{array}{c}
n-1+i \\
i
\end{array}\right)\left(\frac{x y}{n^{2}}\right)^{i}\left(I+\left(\frac{x y}{n^{2}}\right) A\right)^{n} A^{i}-\sum_{i=0}^{\infty}(x y)^{i}(i !)^{-2} A^{i} p\right\| \\
& \leqslant\left\|\sum_{i=0}^{N_{1}}\left[\left(\begin{array}{c}
n-1 \\
i
\end{array}\right)\left(\begin{array}{c}
n-1+i \\
i
\end{array}\right) n^{-2 i}-(i !)^{-2}\right](x y)^{i} A^{i} p\right\| \\
& \text { (20) }+\left\|\sum_{i=0}^{N_{1}}\left(\begin{array}{c}
n-1 \\
i
\end{array}\right)\left(\begin{array}{c}
n-1+i \\
i
\end{array}\right)\left(\frac{x y}{n^{2}}\right)^{i}\left[\left(I+\left(\frac{x y}{n^{2}}\right) A\right)^{n} A^{i} p-A^{i} p\right]\right\| \\
& +\left\|\sum_{i=N_{1}+1}^{n-1}\left(\begin{array}{c}
n-1 \\
i
\end{array}\right)\left(\begin{array}{c}
n-1+i \\
i
\end{array}\right)\left(\frac{x y}{n^{2}}\right)^{i}\left(I+\left(\frac{x y}{n^{2}}\right) A\right)^{n} A^{i} p\right\| \\
& +\left\|\sum_{i=N_{1}+1}^{\infty}(x y)^{i}(i !)^{-2} A^{i} p\right\| \text {. }
\end{aligned}
$$

Reducing this inequality and applying (15), (16), (17) and (18) yields $\| U_{n}(x, y)-$ $U(x, y) \|<\epsilon$. Therefore, $\left\{U_{n}\right\}_{n=1}^{\infty}$ converges uniformly on $[0,1] \times[0,1]$ to the function $U$ defined by equation (14). Since $U$ is a solution to system (II), the proof of Theorem 1 is complete.

III. An implicit product integral representation. In this section, the additional assumption that the operator $\boldsymbol{A}$ generates a strongly continuous semigroup of bounded linear transformations will be used to represent a solution to system (II) as an implicit type of a product integral. The underlying approximation for the implicit technique arises by replacing the integral in equation (1) of the last section by $(x-a)(y-b) A U(x, y)$. Substituting this into equation (1) produces

$$
[I-(x-a)(y-b) A] U(x, y) \approx U(x, b)+U(a, y)-U(a, b) .
$$

The assumption that $A$ generates a strongly continuous linear semigroup implies 
that $(I-\propto A)^{-1}$ exists and is a continuous linear transformation defined on $X$ for all numbers $\alpha$ in $[0, \beta]$ for some positive number $\beta$. This may be used in (21) to give

$$
U(x, y) \approx(I-(x-a)(y-b) A)^{-1}[U(x, b)+U(a, y)-U(a, b)]
$$

for all numbers $x, y, a$ and $b$ such that $0 \leqslant a \leqslant x \leqslant 1,0 \leqslant b \leqslant y \leqslant 1$ and $(x-a)(y-b) \leqslant \beta$. In a process analogous to that of $\S$ II, the approximation given by (22) will be used to construct a sequence of implicit product integral approximations to a solution of system (II).

For each point $(x, y)$ in $[0,1] \times[0,1]$ and each pair $(k, l)$ of positive integers such that $(k l)^{-1} \leqslant \beta$, let $\left\{x_{i k}\right\}_{i=1}^{k},\left\{y_{j l}\right\}_{j=1}^{l}$ and $G_{k l}^{x y}$ be defined as in $\S$ II. Let $\left[W_{k l}^{x y}\right]$ denote a function modeled after $(22)$ and defined on $G_{k l}^{x y}$ by the rule

$$
\left[W_{k l}^{x y}\right]\left(x_{i k}, y_{j l}\right)= \begin{cases}p & \text { if } x_{i k} \cdot y_{j l}=0, \\ {\left[C_{k l}^{x y}\right]\left[\Delta W_{k l}^{x y}\right]\left(x_{i k}, y_{j l}\right)} & \text { if } x_{i k} \cdot y_{j l}>0\end{cases}
$$

where

$$
\left[C_{k l}^{x y}\right]=(I-(x y / k l) A)^{-1}
$$

and

$$
\begin{aligned}
{\left[\Delta W_{k l}^{x y}\right]\left(x_{i k}, y_{j l}\right)=} & {\left[W_{k l}^{x y}\right]\left(x_{i-1 k}, y_{j l}\right)+\left[W_{k l}^{x y}\right]\left(x_{i k}, y_{j-1 l}\right) } \\
& -\left[W_{k l}^{x y}\right]\left(x_{i-1 k}, y_{k-1 l}\right)
\end{aligned}
$$

Let $\omega$ denote a positive integer greater than $\beta^{-1 / 2}$. For each integer $n>\omega$, let $T_{n}$ denote the function defined on $[0,1] \times[0,1]$ by

$$
T_{n}(x, y)=\left[W_{n n}^{x y}\right]\left(x_{n n}, y_{n n}\right)=\left[W_{n n}^{x y}\right](x, y)
$$

An induction type argument on (23), followed by a rearrangement of terms, produces

$$
T_{n}(x, y)=\sum_{i=0}^{n-1}(-1)^{n-1-i}\left(\begin{array}{c}
n-1 \\
i
\end{array}\right)\left(\begin{array}{c}
n-1+i \\
i
\end{array}\right)\left(I-\left(\frac{x y}{n^{2}}\right) A\right)^{-n-i} p
$$


for each integer $n>\omega$ and each point $(x, y)$ in $[0,1] \times[0,1]$.

The following lemma will be used below in Theorem 2 to prove that the sequence $\left\{T_{n}\right\}_{n=\omega}^{\infty}$ of implicit product integral type approximations (defined by (27)) converges uniformly on $[0,1] \times[0,1]$ to a solution of system (II).

LEMMA 3. Suppose $A$ is an infinitesimal generator of a strongly continuous semigroup of bounded linear transformations on the Banach space $X$. Then for each $\epsilon>0$ and each point $q$ in $D(A)$, there is a positive integer $N$ such that

$$
\left\|\left(I-\left(\propto / n^{2}\right) A\right)^{-2 n+1} q-q\right\|<\epsilon
$$

for all $\propto$ in $[0,1]$ and all integers $n \geqslant N$.

Proof. The Hille-Yosida-Phillips theorem (see [4, p. 95]) implies that there is a constant $M>0$ and an integer $N$ such that

$$
\left\|\left(I-\left(\propto / n^{2}\right) A\right)^{-2 n+1}\right\|<M
$$

for all integers $n \geqslant N$ and all numbers $\propto$ in $[0,1]$. The proof of the lemma then follows from the fact that for each point $q$ in $D(A)$ one has that

$$
\begin{aligned}
\left\|\left(I-\left(\propto / n^{2}\right) A\right)^{-2 n+1} q-q\right\| \\
\quad=\left\|\int_{0}^{1} \frac{d}{d t}\left\{\left(I-\left(\propto t / n^{2}\right) A\right)^{-2 n+1} q\right\} d t\right\| \\
=\left\|\int_{0}^{1}\left((2 n-1) \propto / n^{2}\right)\left(I-\left(\propto t / n^{2}\right) A\right)^{-2 n} A q d t\right\| \\
\leqslant(2 / n) M\|A q\|
\end{aligned}
$$

for all $\propto$ in $[0,1]$ and all integers $n>N$.

THEOREM 2. Suppose $A$ is the infinitesimal generator of a strongly continuous semigroup of bounded linear transformations on the Banach space $X$ and $p$ is a point in $D(A)$ which satisfies condition (I). For each positive integer $n>\omega$, let $T_{n}$ be the function defined on $[0,1] \times[0,1]$ by equation (26). Then the sequence $\left\{T_{n}\right\}_{n=\omega}^{\infty}$ converges uniformly on $[0,1] \times[0,1]$ to a solution of system (II).

Proof. Let $\epsilon>0$. From the proof of Lemma 3, let $M>0$ and $N_{1}$ be a positive integer such that $(28)$ is satisfied for all $\propto$ in $[0,1]$ and all integers $n>N_{1}$. The assumption that the point $p$ satisfies condition (I) implies that there exists a positive integer $N_{2}$ such that 


$$
\sum_{i=n}^{\infty}(i !)^{-2}\left\|A^{i} p\right\|<\frac{\epsilon}{4 M+4}
$$

for all integers $n>N_{2}$. Finally, using Lemma 3 and the fact that the point $p$ satisfies condition (I), let $N_{3}\left(>N_{2}\right)$ be a positive integer such that

$$
\left\|\left(I-\left(x y / n^{2}\right) A\right)^{-2 n+1} A^{i} p-A^{i} p\right\|<\epsilon /\left(4 N_{2}\right)
$$

for all integers $n>N_{3}$, all points $(x, y)$ in $[0,1] \times[0,1]$ and all integers $i$ in $\left[0, N_{2}\right]$. Moreover, $N_{3}$ may be chosen such that

$$
\left|1-\left[1-\left(N_{2} / n\right)\right]^{2 N_{2}}\right|<\epsilon /(4 \Delta+4)
$$

for all integers $n>N_{3}$ where $\Delta=\Sigma_{i=0}^{N_{2}}(i !)^{-2}\left\|A^{i} p\right\|$. Let $N=\max \left\{N_{1}, N_{3}\right\}$. Suppose $n$ is an integer greater than $N$ and $(x, y)$ is a point in $[0,1] \times[0,1]$. A rearrangement of the defining equation for $T_{n}$ yields

$$
T_{n}(x, y)=\sum_{i=0}^{n-1}\left(\begin{array}{c}
n-1 \\
i
\end{array}\right)^{2}\left(\frac{x y}{n^{2}}\right)^{i}\left(I-\left(\frac{x y}{n^{2}}\right) A\right)^{-2 n+1} A^{i} p .
$$

From equations (14) and (32) one has that

$$
\begin{aligned}
& \left\|U(x, y)-T_{n}(x, y)\right\| \\
& =\left\|\sum_{i=0}^{\infty}(x y)^{i}(i !)^{-2} A^{i} p-\sum_{i=0}^{n-1}\left(\begin{array}{c}
n-1 \\
i
\end{array}\right)^{2}\left(\frac{x y}{n^{2}}\right)^{i}\left(I-\left(\frac{x y}{n^{2}}\right) A\right)^{-2 n+1} A^{i} p\right\| \\
& \leqslant\left\|\sum_{i=0}^{N_{2}}(x y)^{i}\left[(i !)^{-2}-\left(\begin{array}{c}
n-1 \\
i
\end{array}\right)^{2}\left(n^{-2 i}\right)\right] A^{i} p\right\| \\
& +\left\|\sum_{i=0}^{N_{2}}\left(\begin{array}{c}
n-1 \\
i
\end{array}\right)^{2}\left(\frac{x y}{n^{2}}\right)^{i}\left[I-\left(I-\left(\frac{x y}{n^{2}}\right) A\right)^{-2 n+1}\right] A^{i} p\right\| \\
& +\left\|\sum_{i=N_{2}+1}^{n-1}\left(\begin{array}{c}
n-1 \\
i
\end{array}\right)^{2}\left(\frac{x y}{n^{2}}\right)^{i}\left(I-\left(\frac{x y}{n^{2}}\right) A\right)^{-2 n+1} A^{i} p\right\| \\
& +\left\|\sum_{i=N_{2}+1}^{\infty}(x y)^{i}(i !)^{-2} A^{i} p\right\| .
\end{aligned}
$$


Simplifying the terms in the above inequality and applying (28), (29), (30) and (31) gives $\left\|U(x, y)-T_{n}(x, y)\right\|<\epsilon$. Thus, the sequence $\left\{T_{n}\right\}_{n=1}^{\infty}$ converges uniformly on $[0,1] \times[0,1]$ to the function $U$ defined by equation (14). Since $U$ is a solution to system (II), the proof is complete.

The following corollary, suggested by the referee, follows from the fact that the solution to system (II) is represented in Theorem 2 as a strong limit of continuous linear operators.

CoROllaRY TO THEOREM 2. Under the assumptions of Theorem 2, the solution to system (II) varies continuously with respect to the point $p$.

IV. Bessel function representations. In the case where $X$ is the complex number field and each of $A$ and $p$ is set equal to 1 , the methods of $\S \S$ II and III yield representations for the $J_{0}$ Bessel function. When $A=p=\mathrm{i}$, an argument similar to that used for Theorem 1 may be used to establish that

$$
\begin{aligned}
J_{0}(x) & =\sum_{i=0}^{\infty}(-4)^{-i}(i !)^{-2} x^{2 i} \\
& =\lim _{n \rightarrow \infty} \sum_{i=0}^{n-1}(-1)^{n-1-i}\left(\begin{array}{c}
n-1 \\
i
\end{array}\right)\left(\begin{array}{c}
n-1+i \\
i
\end{array}\right)\left(1-(x / 2 n)^{2}\right)^{n+i}
\end{aligned}
$$

for all real numbers $x$. The corresponding result from $\S$ III is that

$$
J_{0}(x)=\lim _{n \rightarrow \infty} \sum_{i=0}^{n-1}(-1)^{n-1-i}\left(\begin{array}{c}
n-1 \\
i
\end{array}\right)\left(\begin{array}{c}
n-1+i \\
i
\end{array}\right)\left(1+(x / 2 n)^{2}\right)^{-n-i}
$$

for all real numbers $x$.

V. An example. Let $X$ denote the Banach space of all uniformly continuous and bounded real functions defined on $[0, \infty)$ where the norm of $X$ is defined by $\|f\|=\sup _{x \geqslant 0}|f(x)|$ for all $f$ in $X$. For each $t \geqslant 0$, let $T_{t}$ denote the transformation from $X$ to $X$ such that $\left[T_{t} f\right](x)=f(t+x)$ for all $x \geqslant 0$. Let $D(A)$ be the dense subset of $X$ which contains the function $f$ if and only if $f$ is differentiable and $f^{\prime}$ belongs to $X$. Then $A$ will denote the transformation defined on $D(A)$ by the rule $A f=f^{\prime}$. In this setting a solution to system (II) would be a real valued function $w$ satisfying

$$
w_{12}(x, y, z)=w_{3}(x, y, z), \quad w(0, y, z)=p(z)=w(x, 0, z)
$$

for all $(x, y, z)$ in $[0,1] \times[0,1] \times[0, \infty)$ where $p$ is a function in $D(A)$ satisfying condition (I). 
The results of $\S$ II imply that a solution $w$ to system (II') may be represented by

$$
\begin{aligned}
w(x, y, z) & =\sum_{i=0}^{\infty}(x y)^{i}(i !)^{-2} p^{(i)}(z) \\
& =\lim _{n \rightarrow \infty} \sum_{i=0}^{n-1}(-1)^{n-1-i}\left(\begin{array}{c}
n-1 \\
i
\end{array}\right) \cdot\left(\begin{array}{c}
n-1+i \\
i
\end{array}\right)\left(1+\left(x y / n^{2}\right) A\right)^{n+i} p(z)
\end{aligned}
$$

for all $(x, y, z)$ in $[0,1] \times[0,1] \times[0, \infty)$.

From a remark by Butzer and Berens $[1$, p. 134] one may obtain

$$
(I-\lambda A)^{-i} p=p+\frac{\lambda i}{(i-1) !} \int_{0}^{\infty} e^{-\lambda t} t^{i-1}\left[T_{t} p-p\right] d t
$$

for all $\lambda>0$. If $\lambda=0,(I-\lambda A)^{-i} p=p$. The results of Theorem 2 then imply that a solution to system (II') may be represented by

$$
w(x, y, z)= \begin{cases}p(z) & \text { if } x \cdot y=0 \\ p(z)+\lim _{n \rightarrow \infty} G_{n}(x, y, z) & \text { if } x \cdot y>0\end{cases}
$$

where

$$
\begin{aligned}
G_{n}(x, y, z)= & \sum_{i=0}^{n-1}(-1)^{n-1-i}\left(\begin{array}{c}
n-1 \\
i
\end{array}\right)\left(\begin{array}{c}
n-1+i \\
i
\end{array}\right)\left(\frac{n^{2}}{x h}\right)^{n+i}((n+i-1) !)^{-1} \\
& \cdot \int_{0}^{\infty} e^{-(t n / x y)} t^{n+i-1}[p(t+z)-p(z)] d t
\end{aligned}
$$

for all $(x, y, z)$ in $[0,1] \times[0,1] \times[0, \infty)$.

A second example, suggested by the referee, provides an illustration of a case where Theorem 1 applies but Theorem 2 does not. Let $X$ denote the Hilbert space of all square-summable real sequences. Let $D(A)$ denote the subset of $X$ which contains the sequence $x$ if and only if $\Sigma_{k=1}^{\infty} k^{2} x(k)^{2}<\infty$ and let $A$ be the transformation defined by $(A x)(k)=k x(k)$ for each positive integer $k$. Then $A$ is closed and linear. Moreover, each sequence in $X$ with finite support satisfies condition (I). An application of the Hille-Yosida-Phillips theorem (see [4, p. 95]) shows, however, that $A$ does not generate a strongly continuous semigroup of bounded linear transformations. Thus, in this case, Theorem 1 applies but Theorem 2 does not. 


\section{REFERENCES}

1. P. L. Butzer and H. Berens, Semi-groups of operators and approximation, Die Grundlehren der math. Wissenschaften, Band 145, Springer-Verlag, New York, 1967. MR 37 \#5588.

2. P. R. Chernoff, Some remarks on quasi-analytic vectors, Trans. Amer. Math. Soc. 167 (1972), 105-113. MR 45 \#4193.

3. J. B. Diaz, On an analogue of the Euler-Cauchy polygon method for the numerical solution of $u_{x y}=f\left(x, y, u, u_{x}, u_{y}\right)$, Arch. Rational Mech. Anal. 1 (1958), 357-390. MR $21 \# 2803$.

4. A. Friedmann, Partial differential equations, Holt, Rinehart and Winston, New York, 1969.

5. I. I. Glick, On an analog of the Euler-Cauchy polygon method for the partial differential equation $u_{x_{1}} \cdots x_{n}=f$, Contributions to Differential Equations 2 (1963), 1-59. MR 26 \#6555.

6. M. Hasegawa, On a property of the infinitesimal generators of semigroups of linear operators, Math. Ann. 182 (1969), 121-126. MR 40 \#3356.

7. D. L. Lovelady, A fixed point theorem, a perturbed differential equation, and a multivariable Volterra integral equation, Trans. Amer. Math. Soc. 184 (1973), 71-83.

8. J. W. Spellmann, Concerning the infinite differentiability of semigroup motions, Pacific J. Math. 30 (1969), 519-523. MR 40 \#3360.

DEPARTMENT OF MATHEMATICS, PAN AMERICAN UNIVERSITY, EDINBURG, TEXAS 78539 